\title{
Control of mean and fluctuating forces on a circular cylinder at high Reynolds numbers
}

\author{
Chuanping Shao · Jianming Wang
}

Received: 4 July 2006 / Revised: 25 October 2006 / Accepted: 8 November 2006 / Published online: 17 March 2007

(C) Springer-Verlag 2007

\begin{abstract}
A narrow strip is used to control mean and fluctuating forces on a circular cylinder at Reynolds numbers from $2.0 \times 10^{4}$ to $1.0 \times 10^{5}$. The axes of the strip and cylinder are parallel. The control parameters are strip width ratio and strip position characterized by angle of attack and distance from the cylinder. Wind tunnel tests show that the vortex shedding from both sides of the cylinder can be suppressed, and mean drag and fluctuating lift on the cylinder can be reduced if the strip is installed in an effective zone downstream of the cylinder. A phenomenon of mono-side vortex shedding is found. The strip-induced local changes of velocity profiles in the near wake of the cylinder are measured, and the relation between base suction and peak value in the power spectrum of fluctuating lift is studied. The control mechanism is then discussed from different points of view.
\end{abstract}

Keywords Control · Vortex shedding .

Circular cylinder - Mean and fluctuating forces .

High Reynolds number

The project supported by the National Natural Science

Foundation of China (10172087 and 10472124).

The English text was polished by Yunming Chen.

\section{Shao $(\bowtie)$}

Institute of Mechanics, Chinese Academy of Sciences,

Beijing 100080, China

e-mail: shaocp2005@yahoo.com.cn

J. Wang

State Key Laboratory for Turbulence and Complex System,

Peking University, Beijing 100871, China

\section{Introduction}

Vortex shedding naturally occurs behind a bluff body. The pressure distribution on the surface changes as vortices shed alternatively from both sides. A periodic force is then generated which may cause vibration of the body. The vortex-induced vibration is dangerous to engineering structures, not only for durable fatigue damage, but also for possible resonance [1]. Other detrimental effects of the vortex shedding include causing increase in resistance [2] and making monotonous noises [3].

Great efforts have been devoted to the control of bluff body wakes and a number of techniques for different engineering purposes have been developed $[4,5]$.

The theoretical base of the control is related to the mechanism of vortex formation. There are different control ideas originated from different models or conjectures of vortex generation:

(1) Control of separation and base pressure. Laboratory observations indicate that a large area of boundary layer separation is necessary to the generation of vortex shedding. Earlier researchers believed that the attraction of negative base pressure was the reason of inwardly curving and spiraling of the separated shear layers [6,7]. Many methods have been developed to control separation and base pressure, such as enhancing momentum exchange with the flow outside the boundary layer to delay separation, setting a moving surface to avoid separation and setting a tunnel to directly connect the front and rear stagnation points to change the base pressure.

(2) Prevention of interactions between the separated shear-layers. Gerrard [8] proposed a model of 
vortex generation to describe the important role of interactions between the separated shear layers. A long splitter plate was introduced to prevent the interactions and suppress vortex shedding [7-10].

(3) Reduction of span-wise correlation. The synchronization in frequency and phase angle of shedding along the span has important influences on the strength of shedding vortex $[11,12]$. Spiral ribs, span-wise wavy surface and other elements have been introduced to enhance three-dimensionality and reduce span-wise correlation of the flow.

(4) Control of global modes. The oscillations of the wake can be classified into a series of global modes and a fundamental mode representing vortex shedding. Active controls of the fundamental mode were successful at low Reynolds numbers $[13,14]$. At higher $R e$, however, higher modes were excited and nonlinear interactions among the excited modes complicated the control greatly [15].

(5) Elimination of absolute instability. Recent advances in weakly non-parallel flow [15-17] showed that a sufficiently large region of absolute instability in the near wake was responsible for global instability and vortex formation. Base bleeding and base suction have been used to eliminate the absolute instability region [15,18-20].

A good method was given by Strykowsky and Sreenivasan in 1990. In the method, a much smaller circular cylinder was inserted into the wake of main circular cylinder. Vortex shedding from both sides of the main body can be suppressed if the small cylinder is installed in a certain region called effective zone. Unfortunately, their results showed that the effective zone shrank as Reynolds number Re increased. Vortex shedding cannot be suppressed if $R e$ is greater than the order of $10^{2}$. Relations of the suppression to Gerrard's model of vortex generation [8] and to absolute and convective instabilities of the modified flow were explored, but not yet established.

In our recent study [22,23], a narrow strip, rather than a small circular cylinder, was introduced to improve the suppression at $R e=3 \times 10^{3}-2 \times 10^{4}$. Vortex shedding from a circular cylinder can be suppressed if the strip is installed in a region quite similar to the effective zone shown in Ref. [21]. Momentum integrations by using measured downstream velocity profiles show that, the total resistance on cylinder and strip can be reduced to a value much smaller than that on cylinder without control.

In the present paper, we will study strip-control of fluid forces on the cylinder and pay attention to the control mechanism.

\section{Experimental setup}

Two wind tunnels in Peking University were used in the experiment. Tunnel I for pressure and hot-wire measurements and tunnel II for flow visualization. The test section of tunnel I was $5 \mathrm{~m}$ long, $1.0 \mathrm{~m}$ high and $1.2 \mathrm{~m}$ wide. It could supply uniform stream of velocity $2-20 \mathrm{~m} / \mathrm{s}$ with turbulence intensity $0.8-1.0 \%$. A circular cylinder of length $1.0 \mathrm{~m}$ and diameter $D=11 \mathrm{~cm}$ located at the center and spanned the height of the test section. As shown in Fig. 1a, a thin strip of width $b$ and length $0.96 \mathrm{~m}$ was introduced as the control element. Four steel rods of diameter $0.5 \mathrm{~cm}$ and length $25 \mathrm{~cm}$ were perpendicularly installed on the surface and distributed at interval $32 \mathrm{~cm}$ along a meridian of the cylinder to fix the strip. The lengthwise margins of the strip were parallel to and coplanar with the cylinder axis. The cylinder could be
Fig. 1 Cylinder, control element and experimental arrangement

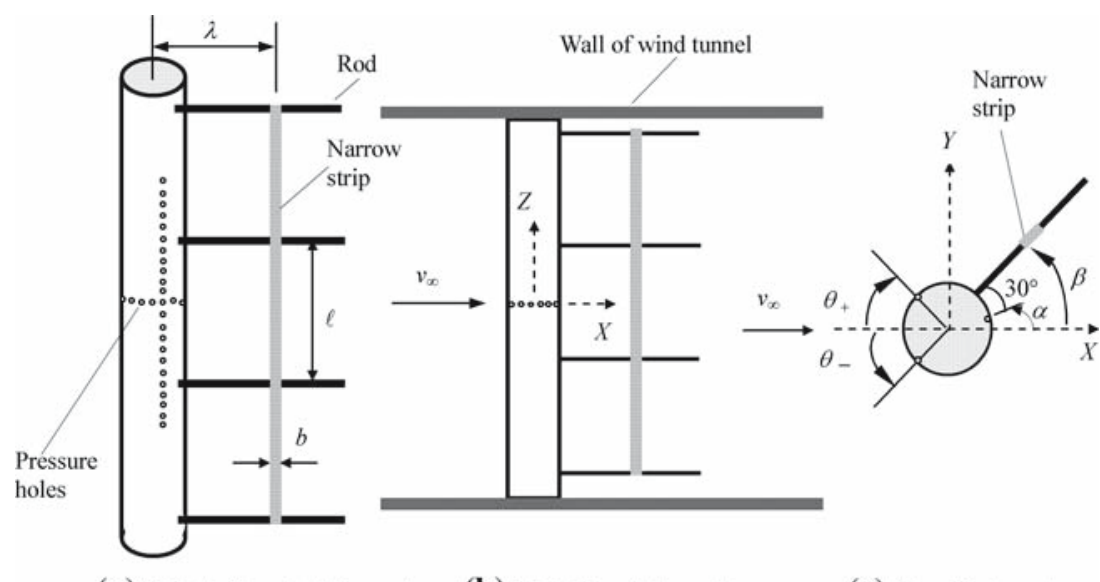

$\begin{array}{lll}\text { (a) Cylinder \& control element } & \text { (b) Model in wind tunnel } & \text { (c) Coordinate system }\end{array}$ 
turned around a short shaft to adjust the angle of attack of the strip $\beta$ within the range $0^{\circ}-90^{\circ}$.

Thirty six pressure holes of diameter $0.12 \mathrm{~cm}$ were set at the mid-span and circumferentially distributed at interval $10^{\circ}$ around the cylinder. Twenty four pressure holes of diameter $0.12 \mathrm{~cm}$ were set on another meridian of the cylinder and distributed at interval $2.0 \mathrm{~cm}$. The azimuth angle between the meridian of rods and the meridian of holes was $30^{\circ}$. Plastic tubes of inner diameter $0.08 \mathrm{~cm}$ and length $0.9 \mathrm{~m}$ were used and set inside the cylinder to connect every pressure hole to a ZOC 33 Electronic Pressure Scanning Module. The pressure variation at each hole was transformed into electric signals by the module and sent by a coaxial cable to the Scanivalve Electronic Scanner System Hyscan 2000 to be recorded. Each tube was fitted with a restrictor at place $35 \mathrm{~cm}$ from the pressure hole to improve its high frequency response. Calibrations were carried out before each test. The sampling frequency and sampling time at each hole were $312 \mathrm{~Hz}$ and $48 \mathrm{~s}$, respectively.

Hot-wire measurements were conducted at station $X / D=7.0$ on the plane of mid-span. Points were measured from $Y / D=-3.5$ to $Y / D=3.5$ at interval $\Delta(Y / D)=0.1-0.25$. The sampling frequency and sampling time were $1,024 \mathrm{~Hz}$ and $30 \mathrm{~s}$, respectively.

Smoke-wire visualization was carried out in tunnel II. The test section was $4 \mathrm{~m}$ long, $0.6 \mathrm{~m}$ high and $0.6 \mathrm{~m}$ wide. It could supply uniform stream of speed $1.0-15.0 \mathrm{~m} / \mathrm{s}$ with turbulence intensity $0.2 \%$. The cylinder was $5 \mathrm{~cm}$ in diameter and $60 \mathrm{~cm}$ in length. The installation of cylinder and strip is similar to that in tunnel I, but six supporting rods were used and the distance between neighboring rods was $11.5 \mathrm{~cm}$. The flow at the section of mid-span was visualized.

The rods have little influence on the flow at the section of mid-span [22]. Vibration of the strip and its influence on flow and aerodynamic characteristics are negligibly small in the present installation.

\section{Results}

The control parameters are strip width $b / D$ and strip position determined by angle $\beta$ and distance $\lambda / D$. The Reynolds numbers tested in hot-wire measurement, pressure measurement and visualization are $2.31 \times 10^{4}-$ $9.24 \times 10^{4}, 6.0 \times 10^{4}-1.0 \times 10^{5}$ and $2.0 \times 10^{4}$, respectively.

\subsection{Effective zones of vortex shedding suppression}

Hot-wire measurements for each strip position have been performed at different points of $Y / D$. The tested strip positions form a net of intervals $\Delta \beta=5^{\circ}$ and $\Delta(\lambda / D)=0.1-0.15$ that covers the area of $0^{\circ} \leq \beta \leq$ $90^{\circ}$ and $0.6 \leq \lambda / D \leq 2.4$. A sharp peak appears in the power spectrum of fluctuating velocity when vortex shedding is not suppressed. On the contrary, the velocity fluctuation is weakened and there appears no outstanding peak in each of the spectra when vortex shedding is suppressed [22]. By such a criterion we can determine the effective zone of strip position. Effective zones corresponding to strip width $b / D=0.18$ with different Reynolds numbers from $2.31 \times 10^{4}$ to $9.24 \times 10^{4}$ and effective zones corresponding to $R e=$ $8.0 \times 10^{4}$ with different $b / D$ widths of strip from 0.136 to 0.272 have been determined and shown in Figs. 2 and 3, respectively. It can be seen from Fig. 2 that there is a

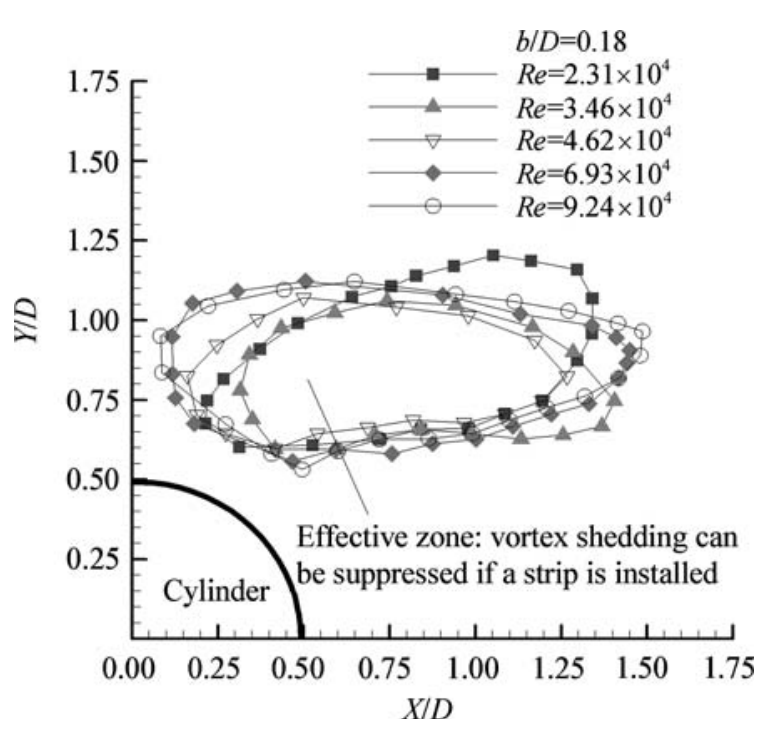

Fig. 2 Effective zones at different Reynolds numbers

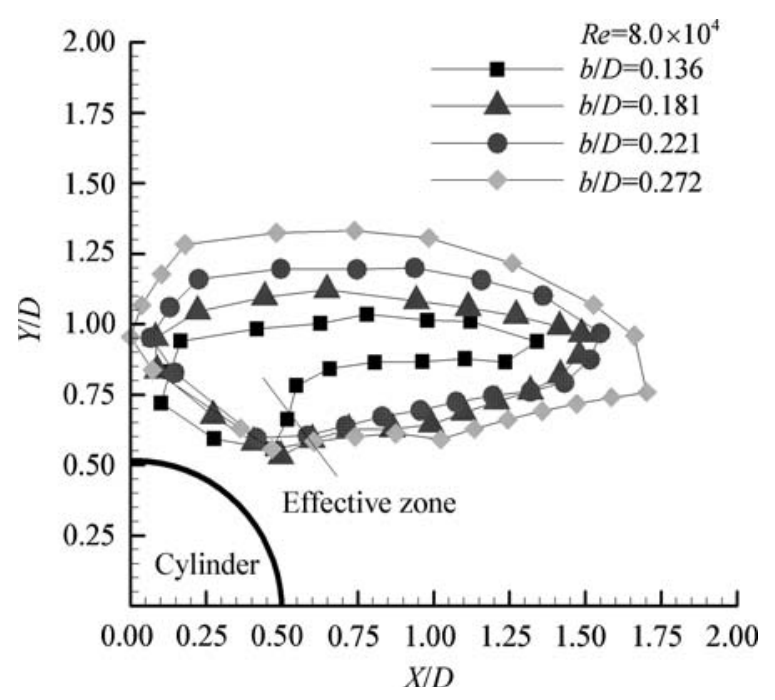

Fig. 3 Effective zones at different strip widths 
considerable overlap area among the effective zones with different Reynolds numbers, which is of practical importance for engineering applications. Figure 3 shows that the size of the effective zone is enlarging with increasing strip width.

\subsection{Suppression of fluctuating lift and drag}

The instantaneous lift and resistance can be obtained by integrating the instantaneous pressure distribution around the cylinder. From the time traces of the integrated forces we can calculate the mean lift and drag coefficients $C_{L}, C_{D}$ as well as the fluctuating lift and drag coefficients $C_{l}(t), C_{d}(t)$.

Figure 4 shows the influence of angle $\beta$ on fluctuating lift $C_{l}(t)$ and its power spectrum at a fixed distance $\lambda / D=1.0$. Large amplitude regular oscillation occurs in the lift without control and a high peak appears at frequency $f D / V_{\infty} \approx 0.2$ in the spectrum. The amplitude of fluctuating lift $C_{l}(t)$ and the peak value in its

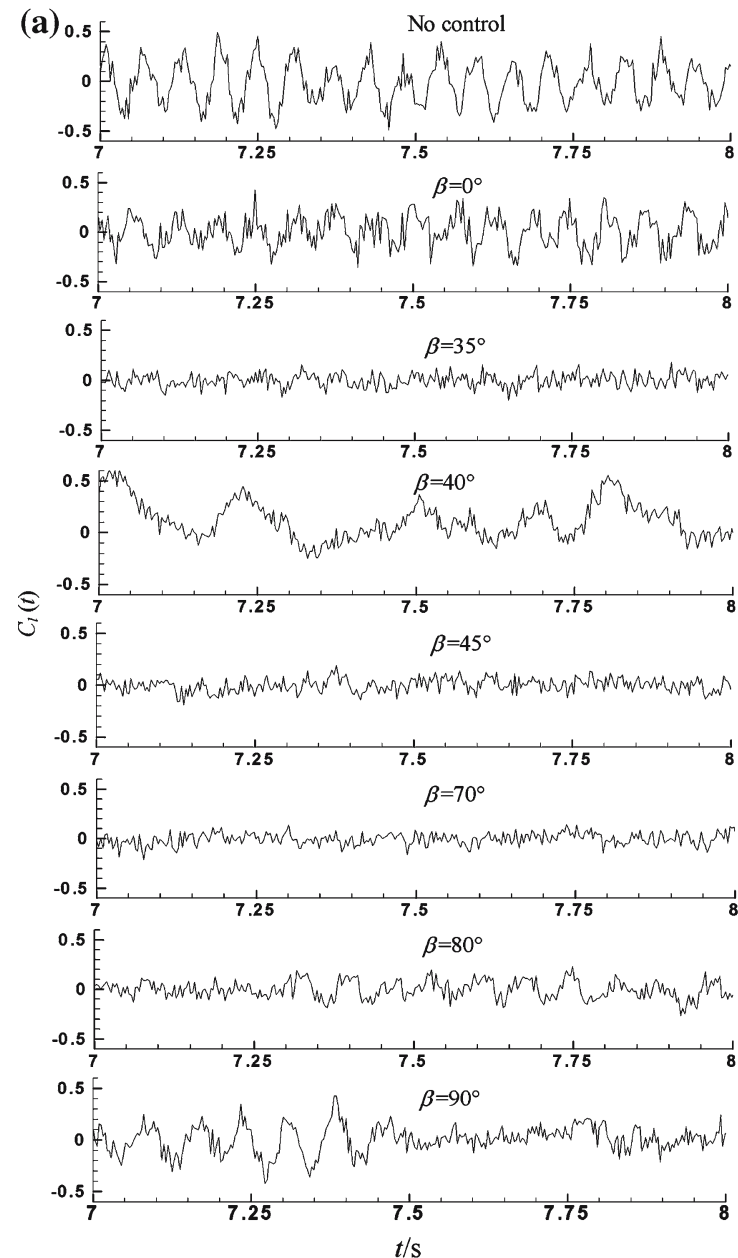

spectrum are reduced by the strip at $\beta=0^{\circ}$ and are continuously decreasing as $\beta$ increases until a threshold angle $40^{\circ}$ is reached. Large amplitude, slowly varying waves appear at this threshold angle, but no outstanding peak is observed in the spectrum. The amplitude of $C_{l}(t)$ is greatly reduced and the sharp peak in the spectrum is removed when the angle is in the range $\beta=45^{\circ}-65^{\circ}$. This is the case where vortex shedding is effectively suppressed. The sharp peak appears again and the peak value increases continuously as $\beta$ increases further from $70^{\circ}$ to $90^{\circ}$, but the peak frequency is notably lower than the one without control. A phenomenon of intermittent switching between large amplitude regular oscillation and small amplitude irregular variation occurs at $\beta=90^{\circ}$. The switching has no great influence on the power spectrum when the sampling time is sufficiently long.

The magnitude of fluctuating resistance can also be reduced in the effective zone, but the rate of reduction is smaller than that of fluctuating lift.

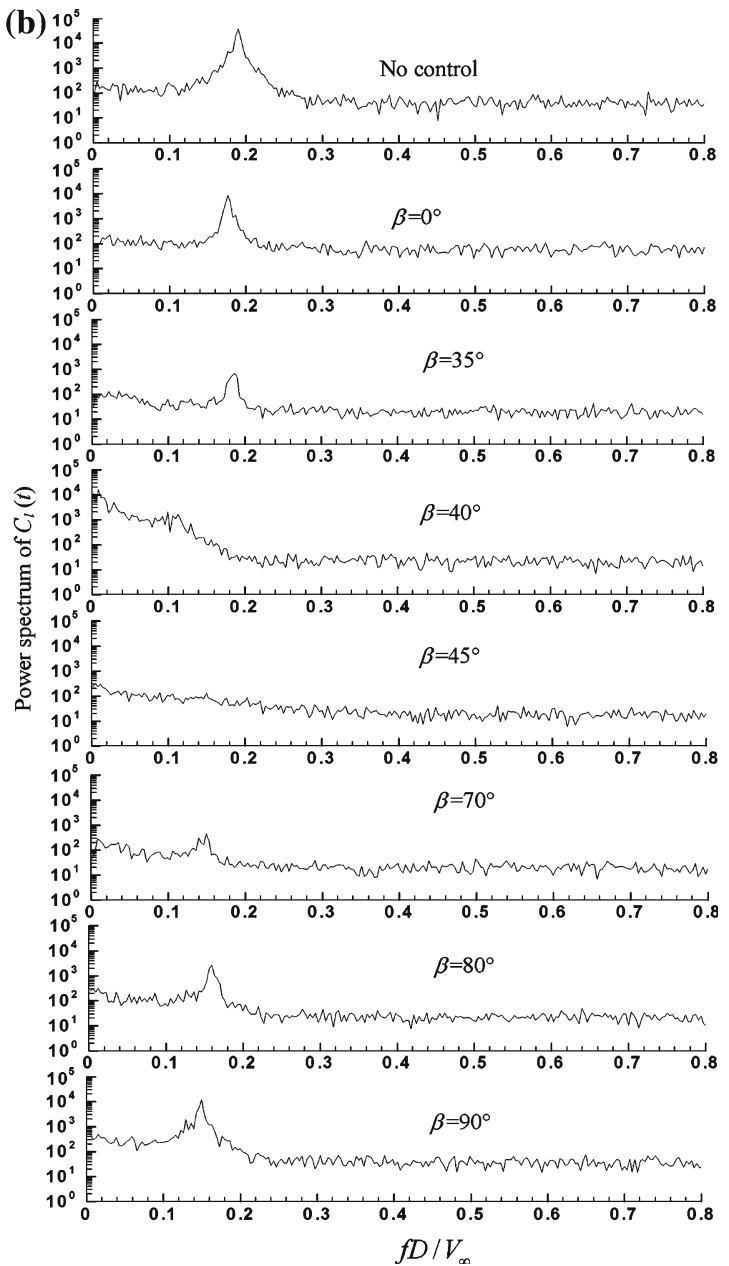

Fig. 4 Influences of angle of attack $\beta$ on fluctuating lift $C_{l}(t)$ and its power spectrum. Strip width $b / D=0.18$, strip distance $\lambda / D=1.0$, $R e=8.0 \times 10^{4}$. a Fluctuating lift $C_{l}(t) ; \mathbf{b}$ Power spectrum of $C_{l}(t)$ 
Fig. 5 Probability density distributions of a magnitude of fluctuating lift Coefficient $C_{l}$ and $\mathbf{b}$ magnitude of fluctuating resistance coefficient $C_{d}$, at fixed distance $\lambda / D=1.0$ and different $\beta$ angles. Strip width $b / D=0.18, R e=8.0 \times 10^{4}$

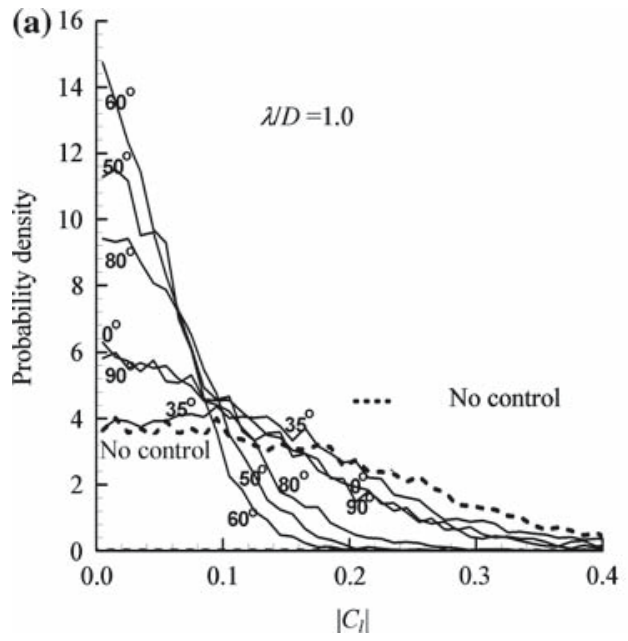

The probability density distributions of the absolute value of fluctuating lift coefficient $\left|C_{l}\right|$ and the absolute value of fluctuating resistance coefficient $\left|C_{d}\right|$, obtained through statistical analysis of the sampling data (time records), are shown in Fig. 5a and b, respectively. The density of $\left|C_{l}\right|$ on cylinder without control is broadly distributed in the range $\left|C_{l}\right|=0-0.4$. There are small or mild changes when the angle changes in the range $\beta=0^{\circ}-35^{\circ}$. A great change occurs as $\beta$ increases across the threshold angle $\left(40^{\circ}\right)$ that the density concentrates rapidly and the main distribution region shrinks quickly towards smaller $\left|C_{l}\right|$. In the range of effective suppression $\beta=45^{\circ}-70^{\circ}$, most of the density is concentrated in the region $\left|C_{l}\right|=0-0.15$. However, the density is continuously diffusing towards larger $\left|C_{l}\right|$ as $\beta$ further increases from $70^{\circ}$, and it is again broadly distributed in the vicinity of $\beta=90^{\circ}$.

Mild changes are found in the probability density distribution of $\left|C_{d}\right|$ when the strip angle $\beta$ changes from $0^{\circ}$ to $90^{\circ}$.

The peak (maximum) value $A$ in the power spectrum of fluctuating lift coefficient represents the portion of fluctuation caused directly by vortex shedding. $A$ is a function of the strip position, and its contour map is shown in Fig. 6 (the value without control is $\lg (A)=4.3$ ). An area that approximately coincides with the effective zone (Fig. 3) occurrs where the $A$ value is reduced by 2 or more orders of magnitude. Only slight changes occur at positions outside the area.

\subsection{Reduction of mean resistance}

The contour map of $C_{D}$ corresponding to varying strip position is shown in Fig. 7 (the value without control is $C_{D}=1.08$ ). An area that covers the effective zone appears where the resistance is reduced by $10-20 \% . C_{D}$ is slightly reduced at other positions.

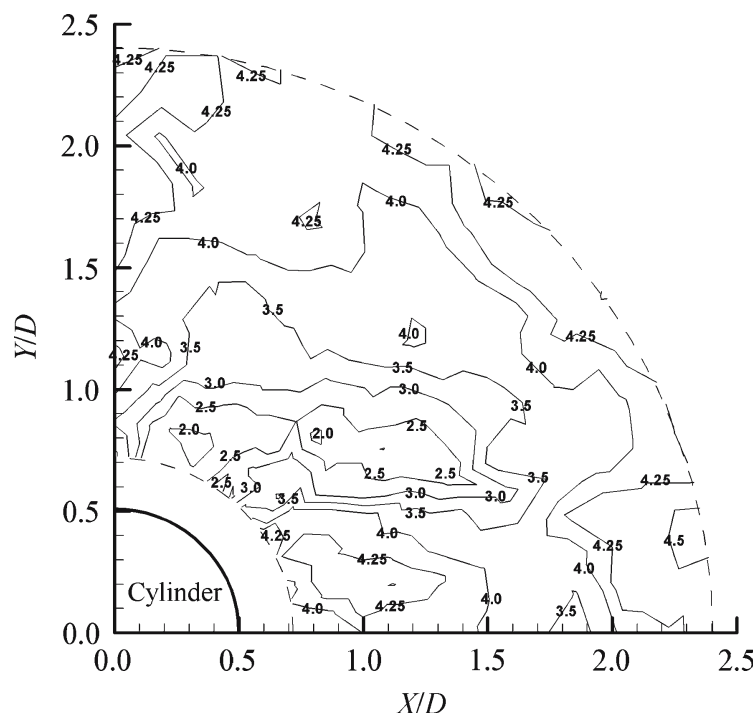

Fig. 6 Contours of $\lg (A)$ with varying strip position, $b / D=0.18$, $R e=8.0 \times 10^{4}$

The $C_{L}$ values in the case of $b / D=0.18$ at $R e=8.0 \times 10^{4}$ are small when the strip is located at any positions except in a small region of $\lambda / D=0.7-0.8$, $\beta=55^{\circ}-65^{\circ}$, where it varies in the range $0.2-0.4$.

All the above results show that, there exists an effective zone of strip position, in which the fluctuating lift acting on the cylinder can be decreased greatly, the mean and fluctuating resistance can be reduced, and the mean lift is kept at a low level.

\section{Discussions}

\subsection{Separation}

Figure 8 shows that the mean pressure distributions around the cylinder without and with suppression 


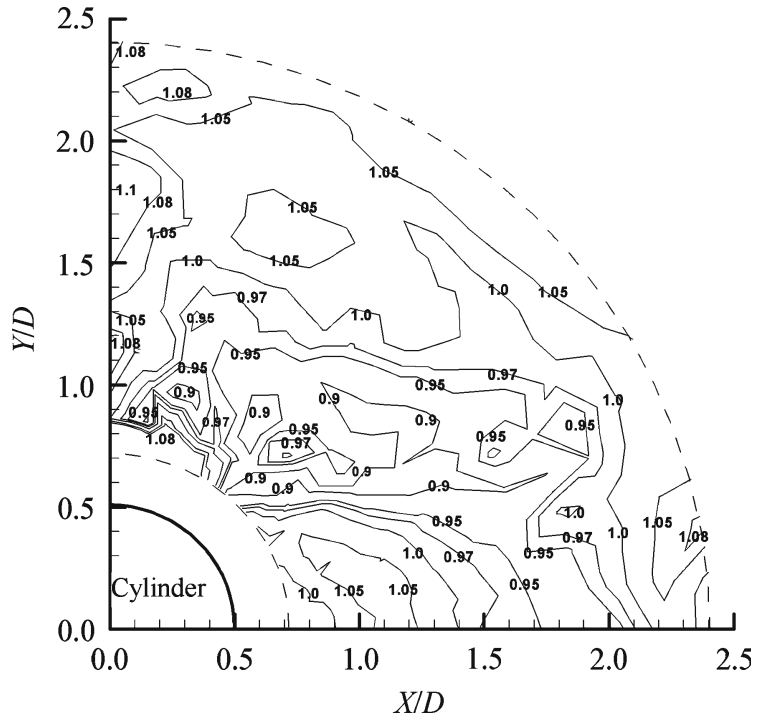

Fig. 7 Contours of mean drag $C_{D}$ with varying strip position, $b / D=0.18, R e=8.0 \times 10^{4}$

(corresponding to the cases in Fig. 4) are of laminar separation pattern. The separation points [24] on both sides of the cylinder are not notably changed by the control. This implies that the present control is different from the separation control.

\subsection{Base suction}

A splitter plate of length $1.0 \mathrm{D}$ or longer has been introduced (set on the wake centerline in Refs.[7] and [9], set parallel to and a distance from the wake centerline in Ref. [10]) to investigate the influence of gap between the cylinder and the plate. Abrupt changes in base suction coefficient $-C_{p b}$, vortex shedding frequency and magnitude of lift fluctuation can be found when the gap exceeds some critical value. In the present study, rapid or abrupt changes in base suction $-C_{p b}$ and Strouhal number $S t$ can also be induced when $\beta$ exceeds a threshold angle (Fig. 9).

The effect of distance $\lambda / D$ of the strip installed on the wake centerline is also studied and shown in Fig. 10. The variations of $A, S t$ and $-C_{p b}$ with $\lambda / D$ are of similar trend, but the data of base suction $-C_{p b}$ are more scattered than those of $A$ and $S t$.

The role of base suction in vortex formation was discussed in Refs. [2,6-10,25]. Wille [6] showed that the negative base pressure region and the inward spiraling of the separated shear layers were correlated. Unal and Rockwell [7] measured the amplitude of fluctuating velocity of the shear layer at $X / D=0.5$, and found that the variation of the amplitude with $R e$ had the same trend as that of base suction coefficient $-C_{p b}$ with $R e$. Roshko [2] showed that the maximum magnitude of fluctuating velocity in the wake increased with increasing $-C_{p b}$.

Bearman [25] and Gerrard [8] introduced a formation length to measure the region of vortex generation. Bearman [25] showed that the formation length was nearly in inverse proportion to the base suction coefficient.
Fig. 8 Mean pressure distributions at different $\beta$ angles, $b / D=0.18$, $\lambda / D=1.0, \operatorname{Re}=8.0 \times 10^{4}$
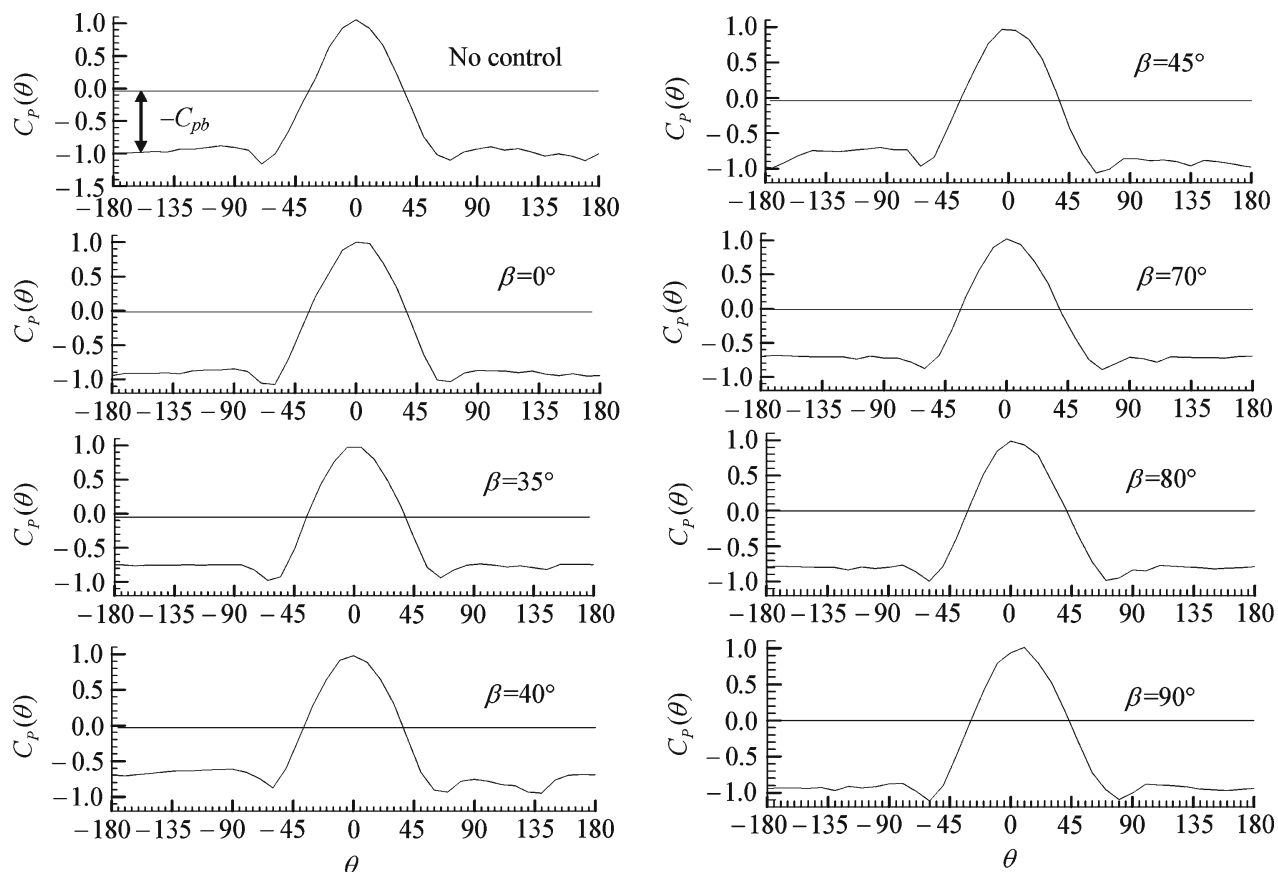
Fig. 9 Changes of a base suction $-C_{p b}$ and $\mathbf{b}$ Strouhal number $S t$ with strip angle $\beta$, $R e=8.0 \times 10^{4}$

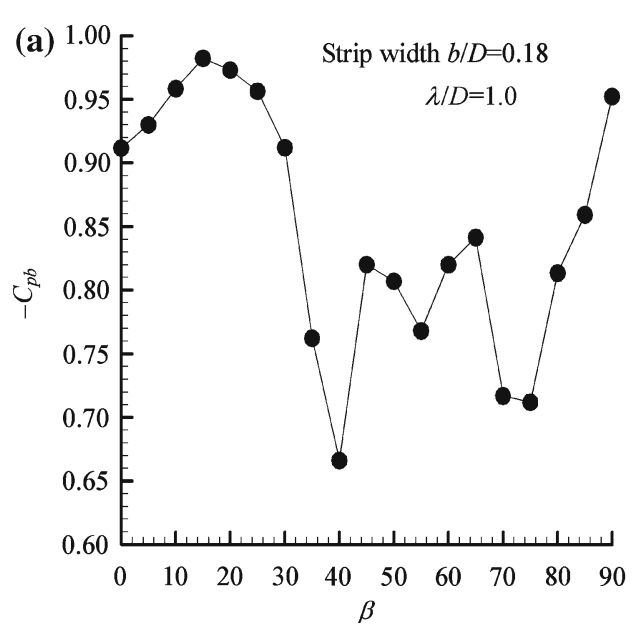

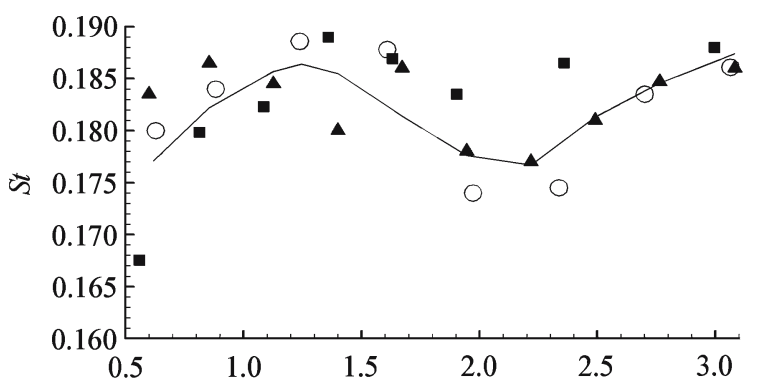
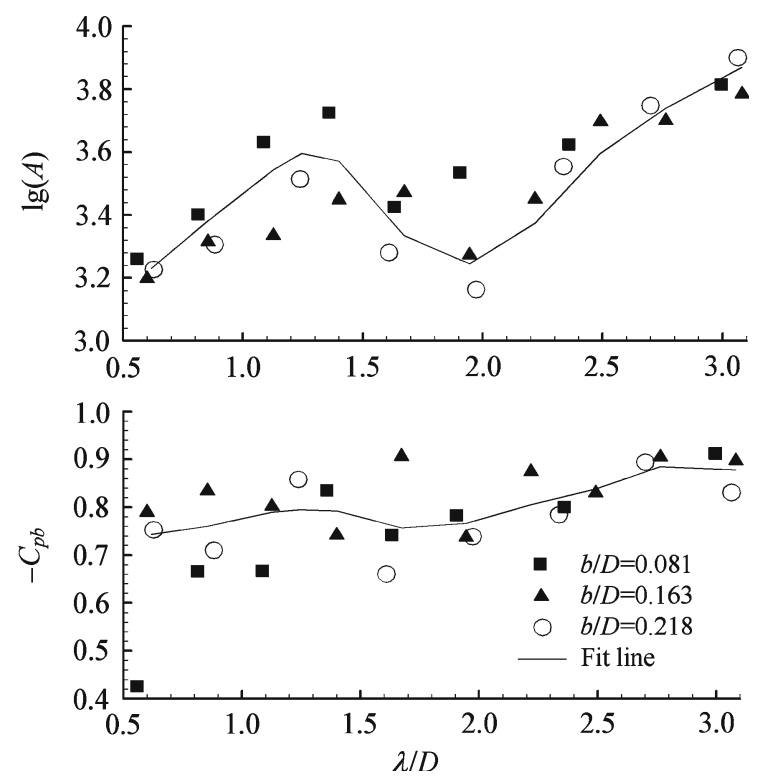

Fig. 10 Influence of strip distance $\lambda / D$ on Strouhal number $S t$, peak value $A$ and base suction, $b / D=0.08-0.22, R e=8.0 \times 10^{4}$

The magnitude of fluctuating lift can be decreased by either decreasing the strength of vortex or increasing the distance between the cylinder and the vortex (i.e. formation length). Unal and Rockwell [7] showed that the increase of formation length and the decrease of vortex strength could be simultaneously achieved by

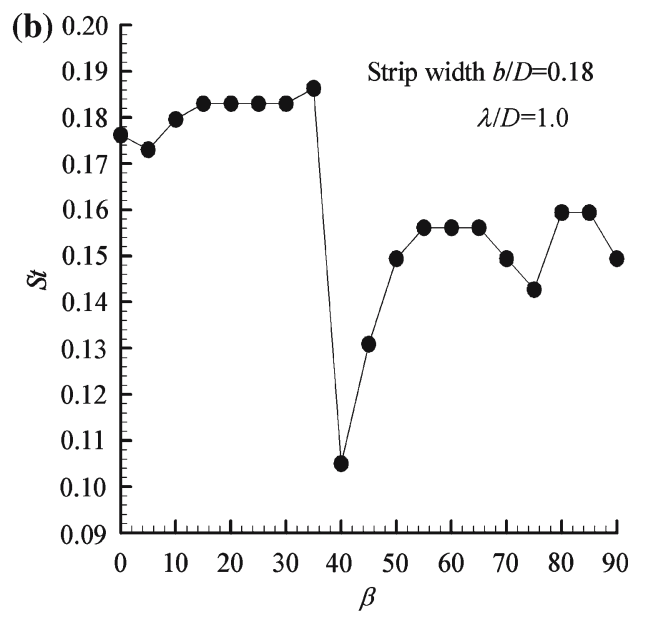

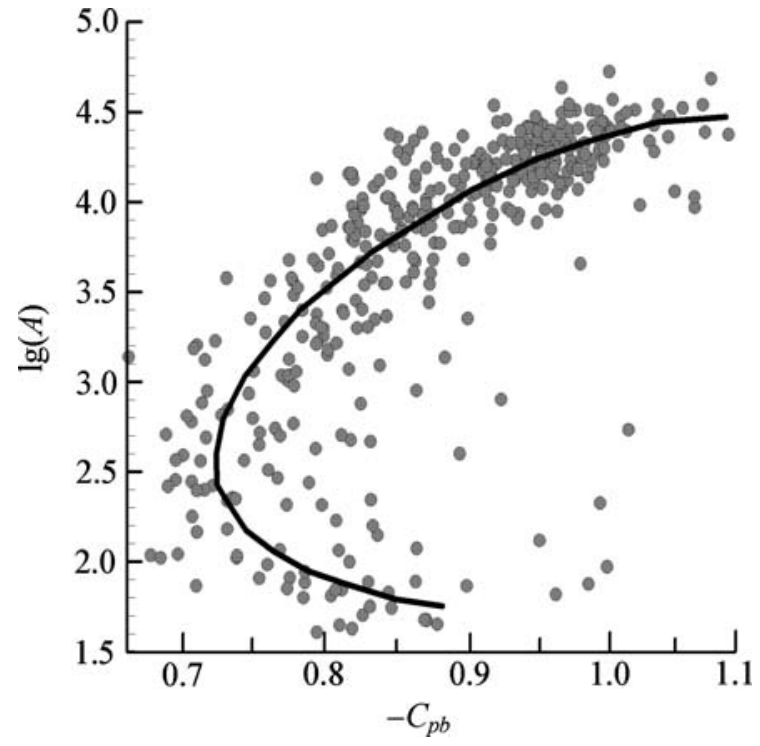

Fig. 11 Statistical correlation between base suction and peak value $A$ in the spectrum of fluctuating lift, $b / D=0.18, R e=$ $8.0 \times 10^{4}$

decreasing the base suction coefficient $-C_{p b}$. So the peak value $A$ in the spectrum of fluctuating lift should be a monotonously increasing function of $-C_{p b}$.

A statistical correlation between the base suction $-C_{p b}$ and the peak value $A$, obtained from data at all measured strip positions, is shown in Fig. 11. The upper part of the fitted line $(\lg (A)>2.5)$ indicates that, the larger $-C_{p b}$, the larger $A$. However, the bifurcation occurred at $\lg (A)=2.5$ and the rather diverged data of $-C_{p b}$ at $\lg (A)<2.5$ indicate a negative fact: $A$ is a multi-value function of $-C_{p b}$, i.e., large value of base suction coefficient $-C_{p b}$ is not sufficient condition for vortex generation. 


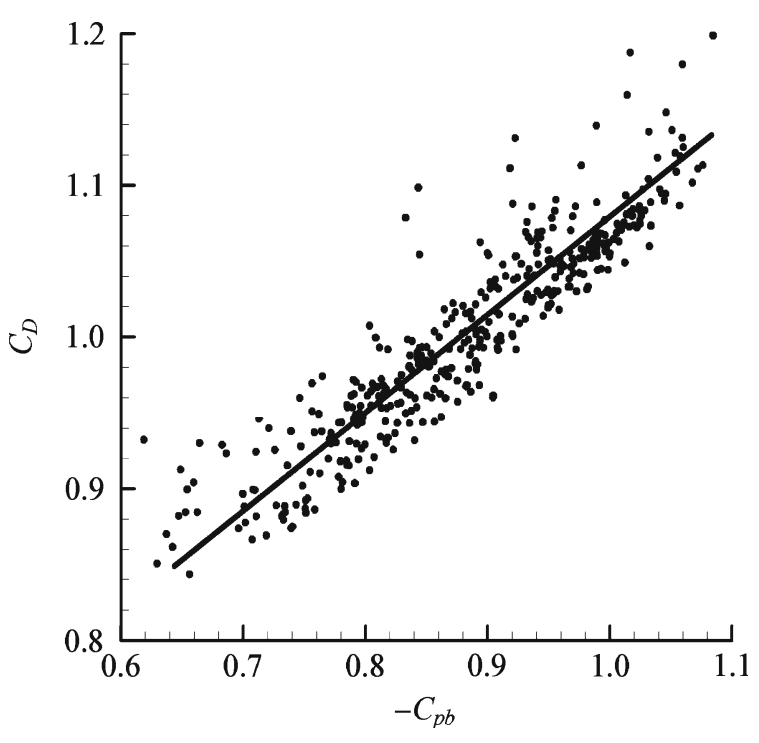

Fig. 12 Statistical correlation between base suction and mean resistance, strip width $b / D=0.18, R e=8.0 \times 10^{4}$

Energy transfer and dissipation in the wake turbulence may play a role in affecting the strength of shedding vortex. The total energy $E$ of the wake flow can be divided into three parts: the part of static pressure $E_{P}$, the part of shedding vortex $E_{A}$ and the part of wake turbulence $E_{T}$. In the cases where vortex shedding is not suppressed, $E-E_{T}$ is divided into $E_{P}$ and $E_{A}$, and the portion of energy $E_{T}$ is not greatly changed. The decrease of $E_{P}$ (i.e., the increase of $-C_{p b}$ ) will increase $E_{A}$ (i.e., increase $A$ ). In the cases where vortex shedding is suppressed or nearly suppressed, $E_{T}+E_{P} \approx E$. The portion $E_{T}$ may change with the strip position, which may induce change in $E_{P}$ and cause divergence in $-C_{p b}$ when $\lg (A)<2.5$.

Statistical analysis of the data measured at all strip positions shows that the mean resistance coefficient $C_{D}$ is nearly proportional to the base suction coefficient $-C_{p b}$ (Fig. 12). It is similar to the relationship exhibited in flows across a bluff flat [2].

\subsection{Span-wise correlation}

The span-wise correlation coefficient can be calculated by

$C(r)=\int_{0}^{T} C_{p}(z, t)^{*} C_{p}\left(z^{\prime}, t\right) \mathrm{d} t / \sqrt{\int_{0}^{T}\left(C_{p}(z, t)^{2} \mathrm{~d} t \int_{0}^{T}\left(C_{p}\left(z^{\prime}, t\right)\right)^{2} \mathrm{~d} t\right.}$, $r=\left|z-z^{\prime}\right| / D$,

where $T$ is sampling time, $C_{p}(z, t)$ and $C_{p}\left(z^{\prime}, t\right)$ are fluctuating pressure coefficients at holes on the meridian (Fig. 1) with coordinate value $z$ and $z^{\prime}$, respectively. The $C(r)$-distributions without and with control are shown in Fig. 13a and b, respectively. It can be seen from Fig. 13a that there are slight differences among the $C(r)$ distributions at different angles of $\alpha$, but all the $C(r)$ values drop dramatically at $r>L \approx 3.2$, where $L$ can be defined as a span-wise correlation length. The value of $L$ here is close to the one shown in Ref. [26]. Compared with the case without control, the $C(r)$ in the suppressed cases (Fig. 13b) are increased slightly. However, the correlation length $L$ is changed little by the suppression.

\subsection{Gerrard's model of vortex generation}

In Gerrard's model [8], the vortex shedding is generated by interactions between the separated shear layers. It was deduced by Strykowsky and Sreenivasan [21] that the function of the small circular cylinder was to diffuse the vorticity in one shear layer. The circulation in the diffused shear layer is then reduced below a threshold, so that the mutual attraction between the opposing
Fig. 13 Comparison of span-wise correlation coefficients with and without control, $R e=8.0 \times 10^{4}$. a Without control; b With control, $b / D=0.18$, $\lambda / D=1.0$
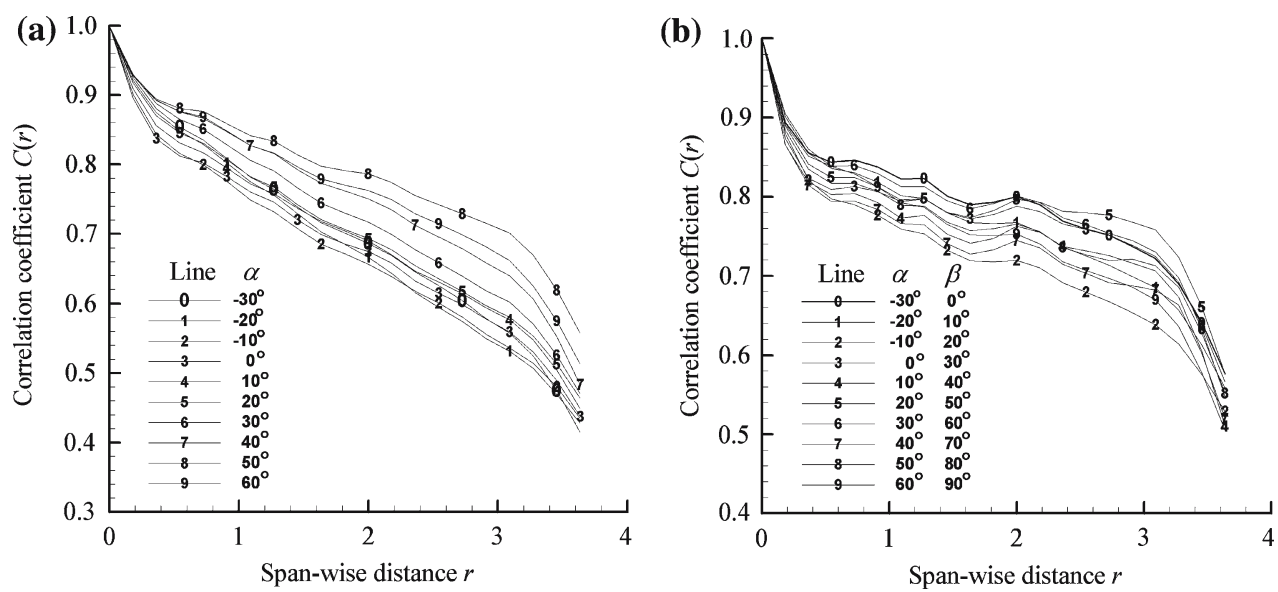
Fig. 14 Effect of strip angle $\beta$ on the control, strip width $b / D=0.18$, strip distance $\lambda / D=1.25, \operatorname{Re}=2.0 \times 10^{4}$

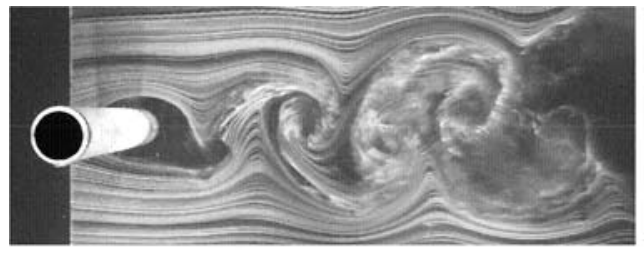

(a) Without control

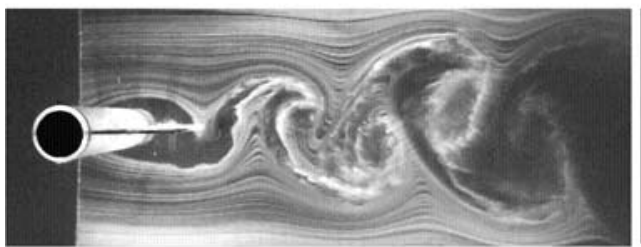

(b) $\beta=0^{\circ}$

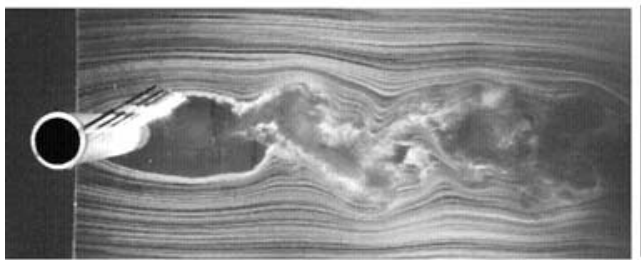

(c) $\beta=35^{\circ}$

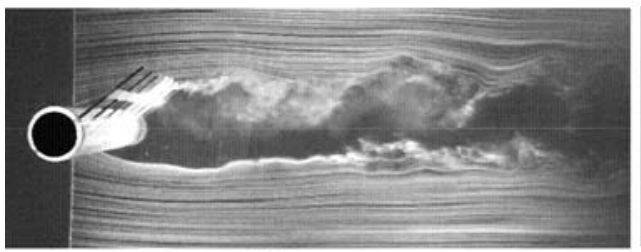

(d) $\beta=45^{\circ}$

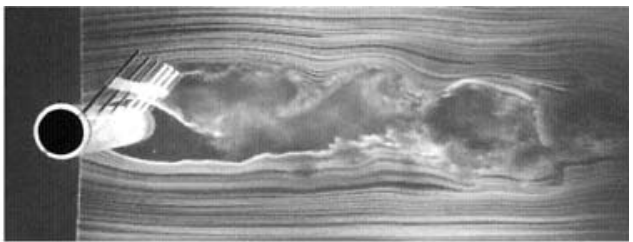

(e) $\beta=55^{\circ}$

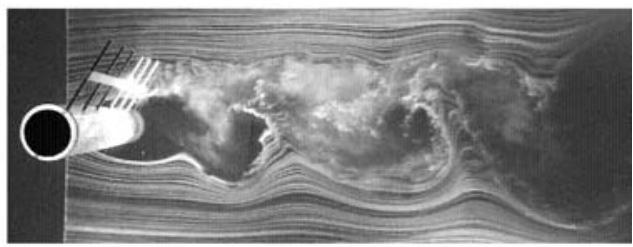

(f) $\beta=65^{\circ}$

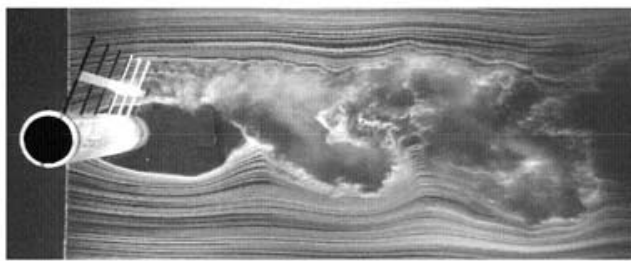

(g) $\beta=75^{\circ}$

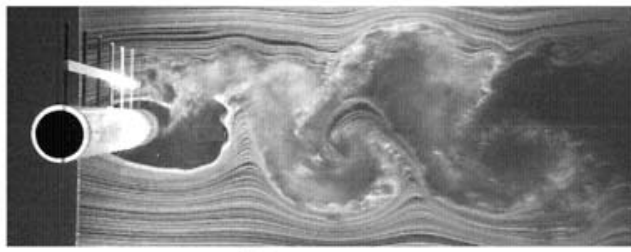

(h) $\beta=90^{\circ}$ shear layers is too weak to form vortex roll-up on both sides. The visualization pictures of Fig. 14d and e show that the "diffusion" effect of the strip can inhibit the "roll-up" of the shear layers on both sides. However, a phenomenon of mono-side vortex shedding is found and shown in Fig. 14f-h, it happens in the range of angle $\beta=60^{\circ}-90^{\circ}$ when $\lambda / D=1.25$. The shear layer on the upper side is diffused and vortex shedding on the diffused side is suppressed, but vortex shedding on the opposite side is still presented.

In the model, the frequency of vortex shedding is also determined by interactions between the separated shear layers. Hot-wire measurements at a downstream station $X / D=12.5$ on the plane of mid-span have been conducted at different $Y / D$ points for the case $\beta=65^{\circ}$, $\lambda / D=1.25$. The results (Fig. 15) show that no obvious peak appears in the power spectrum of fluctuating velocity at each $Y / D$ point on the upper side $(Y / D>0)$ of the wake. However, a sharp peak appears in each spectrum on the lower side $(Y / D<0)$. The peak frequency in each spectrum is $f D / V_{\infty} \approx 0.2$. This means that the frequency of mono-side vortex shedding is nearly the same as that of double-sides vortex shedding without control.

The phenomenon of mono-side vortex shedding is a negative example to Gerrard's model [8] and Strykowsky and Sreenivasan's deduction [21]. More detailed work is needed to resolve the contradiction.

\subsection{Changes of velocity profiles in the near wake}

The properties of bluff body wakes have been investigated by introducing the concepts of global instability and local absolute and convective instability. Stability analyses [27-29] showed that, there was a region in the near wake, where the velocity profiles were absolutely unstable. Possible connections among local absolute instability, temporally growing global modes and vortex shedding were explored in Ref. [21], but not yet established. Later advances in stability theory of weakly non-parallel flow $[16,17]$ indicated that, a sufficiently large region of absolute instability was responsible for global instability and vortex generation. As shown in 


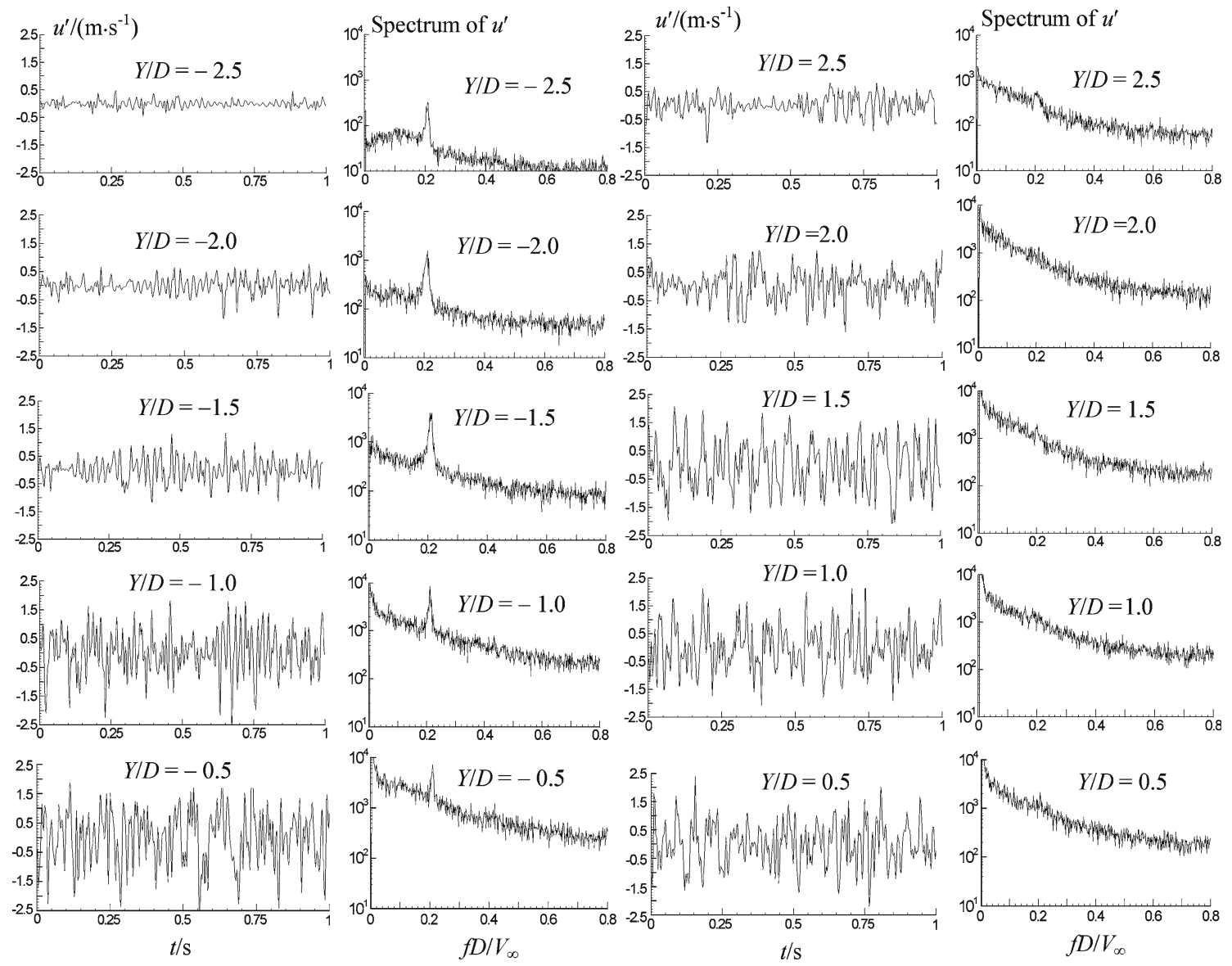

Fig. 15 Fluctuating velocities and their power spectra at different $Y / D$ points on the section $X / D=12.5$. Strip width $b / D=0.18$, $\lambda / D=1.25, \beta=65^{\circ}, R e=2.0 \times 10^{4}$

Fig. 16 Comparison of velocity profiles measured at different $X$-stations in the near wake of a circular cylinder with and without control, $R e=3.85 \times 10^{4}$. a Velocity profiles behind a cylinder without control; b Velocity profiles behind a cylinder controlled by a strip of $b / D=0.18$ placed at $\lambda / D=1.09, \beta=50^{\circ}$
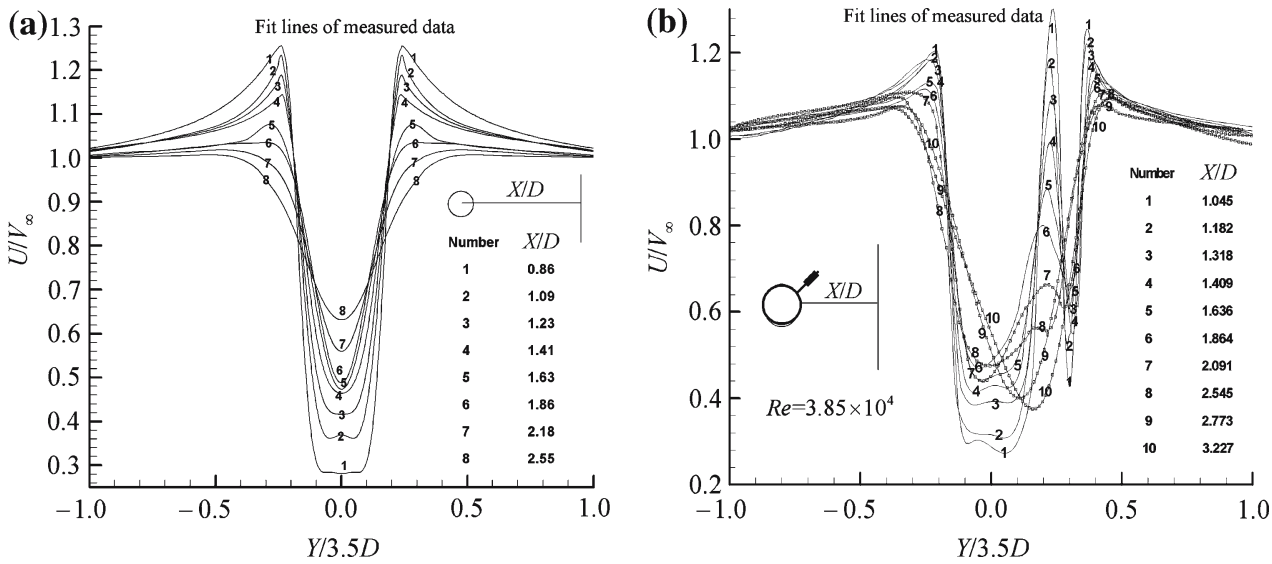

Fig. 16a, the velocity profiles in the near wake of a circular cylinder without control are symmetrical to the wake centerline. They are altered locally by the presence of the strip and the velocities are asymmetrically distributed on the upper $(Y>0)$ and lower $(Y<0)$ sides (Fig. 15b). The local changes of the profiles may induce changes in their stability nature. We may deduce that, when the strip is installed in the effective zone, the absolute instability region in the wake is eliminated or reduced to sufficiently small, so that large scale vortex shedding cannot generate, but it has to be proved by further studies. 


\section{Conclusions}

A narrow strip has been used to control the mean and fluctuating forces acting on a circular cylinder at Reynolds numbers in the range of $R e=2.0 \times 10^{4}-1.0 \times 10^{5}$. The axes of the strip and cylinder are parallel, the control parameters are strip width $b / D$ and strip position determined by angle of attack $\beta$ and distance from the cylinder $\lambda / D$. Wind tunnel tests show that vortex shedding from both sides of the cylinder can be suppressed and the mean drag and fluctuating lift on the cylinder can be reduced if the strip is installed in an effective zone downstream of the cylinder. Effective zones corresponding to $b / D=0.18$ with different Reynolds numbers from $2.31 \times 10^{4}$ to $9.24 \times 10^{4}$ and effective zones corresponding to $R e=8.0 \times 10^{4}$ with different $b / D$ widths from 0.136 to 0.272 are determined. The variation of base suction coefficient $-C_{p b}$ with strip position is studied and abrupt changes are found. A statistical relation between base suction coefficient and magnitude of vortex-induced lift fluctuation is given. A phenomenon of mono-side vortex shedding is observed, and strip induced local changes of velocity profiles in near wake of the cylinder are measured. The control mechanism has been discussed from different points of view.

Acknowledgments The authors thank Prof. Tianfeng Sun and Prof. Qingding Wei for helpful discussions. The authors also thank Dr. Kai Chen, Mr. Yali Wang and Senior Engineer Bin Liang for their helps in preparation of the experiments.

\section{References}

1. Williamson, C.H.K., Govardhan, R.: Vortex-induced vibration. Ann. Rev. Fluid Mech. 36, 413-455 (2004)

2. Roshko, A.: Perspectives on bluff body aerodynamics. J. Wind Eng. Industr. Aerodyn. 49, 79-100 (1993)

3. You, D., et al.: Control of flow induced noise behind a circular cylinder using splitter plates. AIAA J. 36(11), 1961-1967 (1998)

4. Zdravkovich, M.M.: Review and classification of various aerodynamic and hydrodynamic means for suppressing vortex shedding. J. Wind Eng. Industr. Aerodyn. 7, 145-189 (1981)

5. Gad-el--Hak, M.: Flow Control-Passive, Active and Reactive Flow Management. Cambridge University Press, Cambridge (2000)

6. Wille, R.: Generation of oscillatory flows. In: Naudascher E. (ed.) Flow Induced Structural Vibration, 1-16, Springer, Berlin (1974)

7. Unal, M.F., Rockwell, D.: On vortex formation from a cylinder, part 2: Control by splitter-plate interference. J. Fluid Mech. 190, 491-512 (1988)
8. Gerrard, J.H.: The mechanics of the formation region of vortices behind bluff bodies. J. Fluid Mech. 25, 401-413 (1966)

9. Roshko, A.: On the wake and drag of bluff bodies. J. Aeronaut. Sci. 22, 124-132 (1955)

10. Ozono, S.: Flow control of vortex shedding by a short splitter plate asymmetrically arranged downstream of a cylinder. Phys. Fluids 11, 2989-2998 (1999)

11. Rebeiro, J.L.D.: Fluctuating lift and its span-wise correlation on a circular cylinder in a smooth and in a turbulent flow: a critical review. J. Wind Eng. Industr. Aerodyn. 40, 179198 (1992)

12. Zdravkovich, M.M.: Flow around Circular Cylinders. vol. I: Fundamental. Oxford University Press (1997)

13. Roussopoulos, K.: Feedback control of vortex shedding at low Reynolds numbers. J. Fluid Mech. 248, 267-296 (1993)

14. Schumm, M. et al.: Self-excited oscillations in the wake of two-dimensional bluff bodies and their control. J. Fluid Mech. 271, 17-53 (1994)

15. Hammond, D., Redekopp, L.: Global dynamics of symmetric and asymmetric wakes. J. Fluid Mech. 331, 236-260 (1997)

16. Monkewitz, P.A., Huerre, P., Chomaz, J.M.: Global linear stability analysis of weakly non-parallel shear flows. J. Fluid Mech. 251, 1-20 (1993)

17. Chomaz, J.M.: Global instabilities in spatially developing flows: non-normality and non-linearity. Ann. Rev. Fluid Mech. 37, 357-392 (2005)

18. Oertel, H. Jr.: Wakes behind blunt bodies. Ann. Rev. Fluid Mech. 22, 539-565 (1990)

19. Hanneman, K., Oertel, H.J.: Numerical simulation of the absolutely and convectively unstable wake. J. Fluid Mech. 199, 55-88 (1989)

20. Leu, T.S., Ho, C.M.: Control of global instability in a non-parallel near wake. J. Fluid Mech. 404, 345-378 (2000)

21. Strykowsky, P.J., Sreenivansan, K.R.: On the formation and suppression of vortex shedding at low Reynolds numbers. J. Fluid Mech. 218, 71-83 (1990)

22. Shao, C.P., Wang, J.M.: Control of vortex shedding at relatively high Reynolds numbers. Chin. J. Theoret. Appl. Mech. 38(2), 153-161 (2006) (in Chinese)

23. Shao, C.P., Wang, J.M., Wei, Q.D.: Visualization study on suppression of vortex shedding from a cylinder. J. Visualization 10(1), 57-64 (2007)

24. Achenbach, E.: Distribution of local pressure and skin friction around a circular cylinder in cross-flow up to $R e=5 \times 10^{6}$. J. Fluid Mech. 34(4), 625-639 (1968)

25. Bearman, P.W.: Investigation of the flow behind a two-dimensional model with blunt trailing edge and fitted with splitterplates. J. Fluid Mech. 21, 241-255 (1965)

26. Norberg, C.: Fluctuating lift on a circular cylinder: review and new measurements. J. Fluids Struct. 17, 57-96 (2003)

27. Koch, W.: Local instability characteristics and frequency determination of self-excited wake flows. J. Sound Vib. 99, 53-83 (1985)

28. Triantafyllou, G.S., et al.: On the formation of vortex streets behind stationary cylinders. J. Fluid Mech. 170, 461-477 (1986)

29. Monkewitz, P.A., Nuygen, L.N.: Absolute instability in the near-wake of two-dimensional bluff bodies. J. Fluids Struct. 1, 165-184 (1987) 